\title{
Anti-inflammatory drugs and variceal bleeding: a case-control study
}

V De Lédinghen, D Heresbach, O Fourdan, P Bernard, M P Liebaert-Bories, J B Nousbaum, A Gourlaouen, M C Becker, D Ribard, P Ingrand, C Silvain, M Beauchant

\begin{abstract}
Background-Non-steroidal anti-inflammatory drugs (NSAIDs) can have severe gastrointestinal effects and cause peptic ulcers to bleed. Acute bleeding from oesophageal varices is a major complication of cirrhosis of the liver.

Aims-To investigate the role, using a casecontrol study, of NSAIDs in first bleeding episodes associated with oesophageal or cardial varices in cirrhotic patients.

Patients/Methods-A structured interview was conducted of 125 cirrhotic patients with bleeding mainly related to oesophageal varices and 75 cirrhotic controls with oesophageal varices who had never bled.

Results-Cirrhotic patients who were admitted for bleeding related to portal hypertension were more likely to have used NSAIDs during the week before the index day (31 of $125(25 \%)$ ) than the cirrhotic controls (eight of $75(11 \%)$; odds ratio $=2.8, p=0.016)$. Use of aspirin alone or combined with other NSAIDs was also more prevalent in the cases (21 of 125 $(17 \%)$ ) than in the controls (three of 75 $(4 \%)$; odds ratio $=4.9, p=0.007)$. Logistic regression analysis showed that NSAID use $(p=0.022$, odds ratio $=2.9,95 \%$ confidence interval $=1.8$ to 4.7$)$ and variceal size $(p<0.001$, odds ratio $=4.0,95 \%$ confidence interval $=1.4$ to 11.5 ) were the only variables independently associated with the risk of bleeding.

Conclusions-Aspirin, used alone or combined with other NSAIDs, was associated with a first variceal bleeding episode in patients with cirrhosis. Given the life threatening nature of this complication, the possible benefit of this treatment should be weighed against the risk shown here. No firm conclusions could be drawn on non-aspirin NSAIDs used alone. (Gut 1999;44:270-273)
\end{abstract}

Keywords: portal hypertension; non-steroidal anti-inflammatory drugs; variceal bleeding; aspirin; cirrhosis

A number of large case-control and cohort studies have incriminated non-steroidal antiinflammatory drugs (NSAIDs) in gastrointestinal bleeding. ${ }^{1-7}$ Patients who take aspirin or non-aspirin NSAIDs are about three times more likely to have serious gastrointestinal problems $^{1}$ and peptic ulcer bleeding ${ }^{6}$ than those who do not. The risk seems to be influenced by age, a previous history of gastrointestinal problems, the dose and type of NSAID, and concomitant consumption of alcohol, steroids, or a combination of aspirin and non-aspirin NSAIDs. ${ }^{13}$ The other adverse gastrointestinal effects of NSAIDs include non-specific ulceration of the small intestinal mucosa, ${ }^{8}$ which may account for bleeding, ${ }^{9}$ and strictures and protein-losing enteropathy. ${ }^{8}$ However, oesophageal lesions have not been conclusively linked to the use of NSAIDs in epidemiological studies.

Acute bleeding from oesophageal varices is a major complication of hepatic cirrhosis. Elevated portal blood flow and portal pressure ${ }^{10}$ are necessary for the appearance of gastrooesophageal varices, while other factors such as the size of the varix, wall thickness, and transmural pressure tend to favour bleeding. ${ }^{10}{ }^{11}$ The role of NSAIDs in bleeding as the result of portal hypertension has not been evaluated, as cirrhotic patients are systematically excluded from case-control and cohort studies. In a recent study we observed that the use of aspirin was more prevalent in cirrhotic patients presenting with a first episode of variceal bleeding than in non-cirrhotic controls. ${ }^{12}$

This multicentre case-control study was carried out to compare anti-inflammatory drug use by cirrhotic patients admitted to hospital with a first bleeding episode associated with oesophageal or cardial varices and in matched hospitalised cirrhotic control patients.

\section{Methods}

PATIENTS

All cirrhotic adults admitted to nine French hospitals between February 1995 and May 1996 with a first bleeding episode, defined as haematemesis, melaena, or bright red rectal bleeding related to portal hypertension were enrolled in this study. The source of bleeding was identified by endoscopy within 12 hours of admission. Patients were not included if emergency endoscopy was not performed or if it identified another source of bleeding not related to portal hypertension, such as reflux oesophagitis, gastric or duodenal ulcer, gastric carcinoma, or any intestinal lesion not related to portal hypertension. Patients were not included in cases of severe encephalopathy or death, even if their relatives were present at the time of admission, in order to avoid bias during collection of data on previous drug intake.

SELECTION OF CONTROLS

Each patient was matched, whenever possible, with one control of the same sex and age (to within five years), who was admitted for the first time to the same department within two weeks of admission of the case, with a history of

Abbreviation used in this paper: NSAID, non-steroidal anti-inflammatory drug.
University Hospital, BP

Accepted for publication 2 September 1998 
Table 1 Baseline characteristics of cases and controls, habitus, and intake of non-NSAID drugs during the week preceding admission

\begin{tabular}{lcc}
\hline Characteristics & Cases $n=125$ & Controls $n=75$ \\
\hline Age (mean (SD)) & $57(11)$ & $55(10)$ \\
Gender (No of men) & $94(75 \%)$ & $57(76 \%)$ \\
Alcoholic cirrhosis & $110(88 \%)$ & $68(91 \%)$ \\
Child-Pugh class (No of patients) & & \\
A & $24(19 \%)$ & $9(12 \%)$ \\
B & $49(39 \%)$ & $31(41 \%)$ \\
C & $52(42 \%)$ & $35(47 \%)$ \\
Variceal size† (No of patients) & $6(5 \%)$ & $32(43 \%)$ \\
1 & $70(57 \%)$ & $30(40 \%)$ \\
2 & $47(38 \%)$ & $13(17 \%)$ \\
3 & & \\
Ascites (No of patients) & $59(47 \%)$ & $21(28 \%)$ \\
1 & $31(25 \%)$ & $20(27 \%)$ \\
2 & $35(28 \%)$ & $34(45 \%)$ \\
3 & $22(18 \%)$ & $12(16 \%)$ \\
Diabetes mellitus (No of patients) & $70(57 \%)$ & $43(57 \%)$ \\
Smoking (No of patients) & $99(80 \%)$ & $61(81 \%)$ \\
Alcohol consumption (No of patients) & $22(18 \%)$ & $7(9 \%)$ \\
$\beta$ blocker use (No of patients) & $24(19 \%)$ & $11(15 \%)$ \\
Acetaminophen use (No of patients) & & \\
\hline
\end{tabular}

${ }^{\star}$ Class A denotes good hepatic function, class B intermediate hepatic function, and class C poor hepatic function.

†Two data missing; grade 1 corresponds to varices which flatten after insufflation, grade 2 to moderate varices remaining after insufflation, and grade 3 to large varices.

$\ddagger$ Grade 1 corresponds to absence of ascites, grade 2 to moderate and grade 3 to severe ascites. NSAID, non-steroidal anti-inflammatory drug.

cirrhosis. Endoscopy was systematically performed to identify oesophageal or cardial varices. Patients were not included as controls if they had current or previous gastrointestinal bleeding, or previous peptic ulceration or perforation.

DATA COLLECTION

The patients and controls were subjected to the same structured interview by a specially trained doctor. A three page questionnaire was used to obtain details of all drug intake, whether prescribed or self administered, during the week preceding either the bleeding episode (patients) or admission (controls), together with various other features including smoking habits, alcohol consumption, the presence of diabetes, previous bleeding episodes, and the cause of the cirrhosis. The part of the interview on drug intake started with questions on headache, arthralgia, myalgia, fever, or any pain that might have warranted the use of aspirin, non-aspirin NSAIDs, or any other drug. Patients were then asked to recall the trade names in a list of the most popular aspirin and non-aspirin NSAID-containing medications. The precise time of consumption was recorded for each drug, together with the dose and route of administration.

\section{STATISTICAL ANALYSIS}

Baseline characteristics were compared by using Fisher's exact test for proportions and the Mann-Whitney non-parametric test for means, both at the $5 \%$ significance level. The prevalence of the exposure variable, defined as aspirin and/or non-aspirin NSAID use, was compared between cases and controls by using Fisher's exact test. Potentially confounding factors, namely age, gender, Child-Pugh class, ${ }^{13}$ variceal size, $\beta$ blocker use, and paracetamol intake, were taken into account in a stratified analysis using the Mantel-Haenzel $\chi^{2}$ test and multivariate logistic regression analysis. The link between the exposure variables and variceal bleeding is expressed as the odds ratio and $95 \%$ confidence interval. Computations were performed using the SAS package version 6.11 for Windows. The study protocol was approved by the local ethics committee.

\section{Results}

During the study period, 187 consecutive cirrhotic patients were admitted because of bleeding related to oesophageal or cardial varices confirmed by emergency endoscopy. In 125 cases, it was the first episode of bleeding, and these patients were enrolled in the study. During the same period, 128 cirrhotic patients with no previous or current variceal bleeding or previous hospital admission were admitted: endoscopy showed no oesophageal or cardial varices in 53 of these patients. Therefore only 75 controls with varices could be enrolled.

Table 1 gives descriptive statistics for the cases and controls. No significant differences were observed between the two groups in terms of age $(p=0.20)$, gender $(p=1)$, the Child-Pugh class $(\mathrm{p}=0.43)$, serum albumin $(\mathrm{p}=0.62)$, prothrombin time $(\mathrm{p}=0.22)$, degree of encephalopathy $(p=0.26)$, presence of diabetes mellitus $(p=0.85)$, alcohol $(p=0.85)$ and tobacco consumption $(\mathrm{p}=1)$, and recent use of $\beta$ blockers $(p=0.15)$ and paracetamol $(p=$ $0.45)$. At endoscopy, oesophageal varices were found to be significantly larger in the patients than in the controls $(p<0.001$, Fisher's exact test). Bleeders using NSAIDs did not differ from those not using NSAIDs $(p=1)$. Ascites was more severe in the control group $(p=0.004)$ and was the main cause of hospital admission in cirrhotic patients without bleeding.

More patients than controls had used NSAIDs during the week before the index day (31 of $125(25 \%) v$ eight of $75(11 \%)$; odds ratio $=2.8, \mathrm{p}=0.016)$. Use of aspirin alone or combined with non-aspirin NSAIDs was also more prevalent in the cases than the controls (21 of $125(17 \%) v$ three of 75 (4\%); odds ratio $=4.9, \mathrm{p}=0.0066)$.

The following variables were introduced in a logistic regression model: overall NSAID use, age, gender, Child-Pugh class, ascites, variceal size, and recent use of $\beta$ blockers. When a backward elimination procedure was applied, only two of these variables reached the level of significance: overall NSAID use $(\mathrm{p}=0.022$; odds ratio $=2.9 ; 95 \%$ confidence interval $=1.8$ to 4.7$)$ and variceal size $(\mathrm{p}<0.001$; odds ratio $=$ $4.0 ; 95 \%$ confidence interval $=1.4$ to 11.5 ; table $2)$. The goodness of fit, $\chi^{2}$, for this model was 41.0 ( $\mathrm{p}<0.001)$. Using the same model, on

Table 2 Prevalence of NSAID use in cases and controls by variceal size at endoscopy

\begin{tabular}{llll}
\hline Variceal size & $\begin{array}{l}\text { Cases (\%) } \\
n=125\end{array}$ & $\begin{array}{l}\text { Controls (\%) } \\
n=75\end{array}$ & Odds ratio \\
\hline Grade 1 & 17 & 13 & 1.4 \\
Grade 2 & 26 & 13 & 2.3 \\
Grade 3 & 23 & 0 & $\infty$
\end{tabular}

When a backward logistic regression procedure was applied, both variceal size (odds ratio $=4.0 ; 95 \%$ confidence interval $=$ 1.4 to $11.5 ; \mathrm{p}<0.001$ ) and adjusted effect of NSAID use (odds ratio $=2.9 ; 95 \%$ confidence interval $=1.8$ to $4.7 ; \mathrm{p}=0.022)$ reached the level of significance.

NSAID, non-steroidal anti-inflammatory drug. 
introduction of the exposure variable (aspirin alone or combined with non-aspirin NSAIDsthat is, excluding those patients who took only non-aspirin NSAIDs), only two variables were significant, aspirin alone or combined with nonaspirin NSAIDs $(\mathrm{p}=0.020$; odds ratio $=4.7$; $95 \%$ confidence interval $=1.3$ to 17.3 ) and variceal size $(\mathrm{p}<0.001$; odds ratio $=3.9 ; 95 \%$ confidence interval $=2.4$ to 6.6$)$. The goodness of fit, $\chi^{2}$, for this model was 42.3 ( $\left.p<0.001\right)$. In patients with grade 1 oesophageal varices, neither NSAID use (odds ratio $=1.4, \mathrm{p}=0.78$ ) nor use of aspirin alone (odds ratio $=6.2, \mathrm{p}=$ 0.22 ) was associated with an increased risk of bleeding.

Among the 125 cases, there were no differences between NSAID users and nonusers in terms of the site of bleeding (oesophageal varices in 30 of 31 users $(97 \%)$ and 91 of 94 non-users $(97 \%)$; cardial varices in one $(3 \%)$ and three $(3 \%)$ respectively; none bled from gastric varices; severe portal hypertensive gastropathy was observed in one non-user). Similarly there were also no differences between NSAID users and non-users in terms of the presence of non-bleeding lesions which were not considered as the cause of bleeding at emergency endoscopy: oesophageal erosions or ulcers (two of $31(6 \%)$ of NSAID users, and six of $94(6 \%)$ of non-users) or gastric and/or duodenal ulcers or erosions (four $(13 \%)$ and $14(15 \%)$ in users and non-users respectively). NSAIDs were used by patients with bleeding for the following main reasons: headache in eight cases, arthralgia in six, myalgia in three, flu-like illness in three, coronary heart disease in four, various pains in three, and other causes in four. Among the 21 patients who used aspirin, five took less than $300 \mathrm{mg}$ per day and 16 took $300 \mathrm{mg}$ or more per day. Aspirin was usually taken in tablet form. Five patients took buffered tablets and none used enteric-coated preparations.

\section{Discussion}

This case-control study suggests that cirrhotic patients who use NSAIDs are about three times more likely to have a first variceal bleeding episode than cirrhotic patients who do not. The risk appeared to be due mainly to aspirin, alone or combined with other NSAIDs, only in patients with grade 2 or 3 varices. No conclusions on exclusively non-aspirin NSAID use or severity of bleeding related to NSAID use could be drawn. To avoid bias related to misinterpretation of endoscopic findings, we chose to enrol patients bleeding from oesophageal or cardial varices. Bleeding from isolated gastric varices was never observed during the study period. Non-bleeding isolated gastric varices or portal hypertensive gastropathy was not systematically recorded in either patients or controls.

Aspirin is usually self administered in France, and non-aspirin NSAIDs were not available over the counter at the time of the study. There is no clear reason for the low prescription rate of non-aspirin NSAIDs, although they may have adverse renal effects in cirrho- sis, ${ }^{14}$ and their known gastrointestinal toxicity in non-cirrhotics may also have reduced their prescription.

Several potential biases must be taken into account in this study. Cirrhotic patients with a previous history of bleeding were not enrolled as they may have avoided NSAIDs. It was difficult to recruit age- and sex-matched cirrhotic patients found to have oesophageal or gastric varices at endoscopy who reported never having bled previously. This was not surprising, as the usual reason for the first hospital admission of patients with medium or large varices is variceal bleeding. As a consequence, variceal size differed between cases and controls, and logistic regression analysis showed that variceal size and NSAIDs were independently associated with bleeding.

This case-control study was unlikely to have suffered from the classical bias related to the prior beliefs of the doctors about NSAIDs and disease, as the interviews with both patients and controls were conducted by the same doctor in each centre using a structured questionnaire. The investigator was not blinded to the subjects' status (case or control), as all patients with gastrointestinal bleeding, whatever the cause, are admitted to a specialised intensive care unit in each of the participating centres. However, the use of another common nonprescription analgesic, paracetamol, was comparable in the two groups, arguing against an overestimation of drug intake in the cases. The link between NSAIDs and bleeding was unchanged after controlling for confounding variables by logistic regression. The severity of the liver disease (Child's class) was comparable in the cases and controls and did not affect the risk of bleeding in the logistic regression model. Patients with confusion related to encephalopathy were not included in order to minimise recall bias, although we cannot exclude the possibility that subclinical encephalopathy was present. However, the same prevalence of paracetamol intake in the two groups argues against such recall bias. The prevalence of recent alcohol intake was also similar in the two groups. The first variceal bleeding episode is easily predicted by variceal size, ${ }^{15}$ and in this study, logistic regression analysis confirmed that variceal size was independently associated with bleeding. The prevalence of NSAID use by the cirrhotic patients who bled was lower than the $34 \%$ that we observed in a previous study, ${ }^{12}$ but similar to the $19 \%$ reported by Wilcox et al. ${ }^{16}$ NSAID use by the cirrhotic controls (11\%) compared well with that $(12 \%)$ in non-cirrhotic controls in a previous study. ${ }^{12}$

Precisely how aspirin induces variceal bleeding cannot be deduced from our results. Rupture of oesophageal varices is mainly due to raised variceal pressure. ${ }^{10}$ Oesophagitis is controversial as a cause of variceal bleeding. Oesophageal transection has been used as a salvage procedure, and careful histological analysis of transection rings provided no evidence of oesophagitis, ${ }^{17}{ }^{18}$ although NSAID use in the few cases reported was not mentioned. However, large subepithelial blood 
filled channels are frequent in the portal hypertensive oesophagus and may indicate increased variceal fragility. ${ }^{18}$ NSAID use is not a risk factor for ulcerative reflux oesophagitis in non-cirrhotics. ${ }^{19}$ Many reports have shown a link between NSAID use and the emergence of oesophageal ulcers, benign strictures, and bleeding, ${ }^{20-26}$ although some authors have suggested that non-aspirin NSAIDs may have protective properties in these respects. ${ }^{27} 28$

NSAID induced gastric mucosal damage probably results from a complex mechanism. Local mucosal injury and inhibition of cyclooxygenase type 1 activity lead to a reduction in mucosal defences, restricted mucosal blood flow, ${ }^{29}$ and gastric erosions, which may facilitate cardial or fundic variceal rupture. However, the prevalence of non-bleeding ulcers and erosions in this study was comparable in NSAID users and non-users. Another possible explanation for the increased risk of bleeding is the inhibitory effect of aspirin on platelet aggregation, ${ }^{30}$ added to the platelet adhesion defect which may already be present in patients with cirrhosis. ${ }^{31}$

In conclusion, aspirin, alone or combined with non-aspirin NSAIDs, was associated with a first variceal bleeding episode in patients with cirrhosis. Given the life threatening nature of this complication, the possible benefit of the drug must be weighed against the risk shown here. No firm conclusions could be drawn on the use of non-aspirin NSAIDs alone. Paracetamol remains the preferred over the counter analgesic and antipyretic drug, as it carries the lowest risk of adverse reactions, even in alcoholics, when used at the recommended doses. $^{32}$

This work was supported in part by grants from the Institut de Recherche sur les Maladies de l'Appareil Digestif (IRMAD) and Astra France Laboratories. The following centres participated in Astra France Laboratories. The following centres participated in the study: CHU Poitiers (M Beauchant, V De Ledinghen, P Ingrand, C Silvain), CHU Rennes (D Heresbach, J F Bretagne), CHU Paris-St Antoine (O Fourdan, C Florent, V G Lévy),
CHU Bordeaux (P Bernard, A Quinton), CHU Limoges (M P CHU Bordeaux (P Bernard, A Quinton), CHU Limoges (M P
Liebaert-Bories, B Pillegand), CHU Brest (I B Nousbaum, H Liebaert-Bories, B Pillegand), CHU Brest (J B Nousbaum, H Gouérou), CH Morlaix (A Gourlaouen), CHU Besançon (
Becker, J P Miguet), CHU Nîmes (D Ribard, D Balmes).

1 Gabriel SE, Jaakkimainen L, Bombardier C. Risk for serious gastrointestinal complications related to use of nonsteroida anti-inflammatory drugs. A meta-analysis. Ann Intern Med 1991;115:787-96.

2 Garcia Rodriguez LA, Jick H. Risk of upper gastrointestinal bleeding and perforation associated with individual nonsteroidal anti-inflammatory drugs. Lancet 1994;343:76972 .

3 Henry D, Dobson A, Turner C. Variability in the risk of major gastrointestinal complications from nonaspirin nonsteroidal anti-inflammatory drugs. Gastroenterology 1993 105:1078-88.

4 Holvoet J, Terriere L, Van Hee W, et al. Relation of upper gastrointestinal bleeding to non-steroidal antiinflammatory drugs and aspirirn: a case-control study. Gut 1991;32:730-4.

5 Laine L, Peterson W. Bleeding peptic ulcer. $N$ Engl f Med 1994;331:717-27.
6 Langman MJS, Weil J, Wainwright $\mathrm{P}$, et al. Risks of bleeding peptic ulcer associated with individual non-steroidal
anti-inflammatory drugs. Lancet 1994;343:1075-8.

7 Laporte JR, Carné X, Vidal X, et al. Catalan countries study on upper gastrointestinal bleeding. Upper gastrointestinal bleeding in relation to previous use of analgesics and nonsteroidal anti-inflammatory drugs. Lancet 1991;337:85-9.

8 Allison MC, Howatson AG, Torrance CJ, et al. Gastrointestinal damage associated with the use of nonsteroidal antiiflammatory drugs. N Engl f Med 1992;327:749-54.

9 Holt S, Rigoglioso V, Sidhu M, et al. Nonsteroidal antiinflammatory drugs and lower gastrointestinal bleeding. Dig Dis Sci 1993;38:1619-23.

10 Garcia-Tsao G, Groszmann RJ, Fisher RL, et al. Portal pressure, presence of gastroesophageal varices and variceal bleeding. Hepatology 1985;5:419-24.

11 Lebrec D, De Fleury P, Rueff B, et al. Portal hypertension, size of esophageal varices, and risk of gastrointestinal bleeding in alcoholic cirrhosis. Gastroenterology 1980;79: 1139-44.

12 De Ledinghen V, Mannant PR, Foucher J, et al. Non steroidal anti-inflammatory drugs and variceal bleeding: a casecontrol study. F Hepatol 1996;24:570-3.

13 Pugh RMH, Murray-Lyon IM, Dawson JL, et al. Transection of the oesophagus for bleeding oesophageal varices. $\mathrm{Br}$ f Surg 1973;60:646-9

14 Wong F, Massie D, Hsu P, et al. Indomethacin-induced renal dysfunction in patients with well-compensated cirrhosis. Gastroenterology 1993;104:869-76.

15 The North Italian Endoscopic Club for the Study and Treatment of Esophageal Varices. Prediction of the first variceal hemorrhage in patients with cirrhosis of the liver and esophageal varices. A prospective multicenter study. $N$ Engl f Med 1988;319:983-9.

16 Wilcox CM, Shalek KA, Cotsonis G. Striking prevalence of over-the-counter nonsteroidal anti-inflammatory drug use in patients with upper gastrointestinal hemorrhage. Arch Intern Med 1994;154:42-6.

17 Ponce J, Froufe A, De la Morena E, et al. Morphometric study of the esophageal mucosa in cirrhotic patients with variceal bleeding. Hepatology 1981;1:641-6.

18 Spence RAJ, Sloan JM, Johnston GW. Histologic factors of the esophageal transection ring as clues to the pathogenesis of bleeding varices. Surg Gynecol Obstet 1984;159:253-9.

19 Ryan P, Hetzel DJ, Shearman DJ, et al. Risk factors for ulcerative reflux oesophagitis: a case-control study. 7 Gastroenterol Hepatol 1995;10:306-12.

20 Bellary SV, Isaacs PET, Lee FI. Upper gastrointestinal lesions in elderly patients presenting for endoscopy: relevance of NSAID usage. Am f Gastroenterol 1991;86: 961-4

21 Heller SR, Fellows IW, OgilvieAL, et al. Non-steroidal antiinflammatory drugs and benign oesophageal stricture. BMF 1982;285:167-8.

22 Lanas A, Hirschowitz BI. Significant role of aspirin use in patients with esophagitis. F Clin Gastroenterol 1991;13:622-7.

23 Semble EL, Wu WC, Castell DO. Nonsteroidal antiinflammatory drugs and esophageal injury. Semin Arthritis Rheum matory drugs and

24 Taha AS, Nakshabendi I, Sturrock RD, et al. Non-steroidal anti-inflammatory drugs and the esophagus: endoscopic and histological study. [Abstract] Gastroenterology 1993; and histologic

25 Wilkins WE, Ridley MG, Pozniak AL. Benign stricture of the oesophagus: role of non-steroidal anti-inflammatory drugs. Gut 1964;25:478-80.

26 Wolfsen HC, Wang KK. Etiology and course of acute bleeding esophageal ulcers. F Clin Gastroenterol 1992;14:342-6.

27 Taha AS, Dahill S, Nakshabendi I, et al. Oesophageal histology in long term users of non-steroidal anti-inflammatory drugs. F Clin Pathol 1994;47:705-8.

28 Eastwood GL, Beck BD, Castelle DO, et al. Beneficial effect of indomethacin on acid-induced esophagitis in cats. Dig Dis Sci 1981;26:601-8.

29 Mahmud T, Scott DL, Bjarnason I. A unifying hypothesis for the mechanism of NSAID related gastrointestinal toxicity. Ann Rheum Dis 1996;55:211-13.

30 Lanas AI, Arroyo MT, Esteva F, et al. Aspirin related gastrointestinal bleeders have an exaggerated bleeding time response due to aspirin use. Gut 1996;39:654-60.

31 Ordinas A, Escolar G, Cirera I, et al. Existence of a plateletadhesion defect in patients with cirrhosis independent of hematocrit: studies under flow conditions. Hepatology 1996;24:1137-42.

32 Strom BL. Adverse reactions to over-the-counter analgesics taken for therapeutic purposes. $7 A M A$ 1994;272:1866-7. 